\title{
The need for a new strategy for Ebola vaccination
}

\author{
News of two new outbreaks of Ebola virus disease in the Democratic Republic of the Congo and Republic of Guinea, \\ on the backdrop of the COVID-19 pandemic, signals the need for a change of direction in vaccination strategies in
} the area.

\section{Daniel G. Bausch}

. ere we go again. In February 2021, two new outbreaks of Ebola virus disease (EVD) were declared, kicking into gear now familiar, but far from perfect, national and international efforts at control. With each outbreak declaration, there is fear of a major disruptive event costing thousands of lives and millions of dollars. And, of course, in the midst of the ongoing COVID-19 pandemic, another deadly viral outbreak is especially unwelcome.

Both outbreaks are in places where large EVD outbreaks have been seen before: the Democratic Republic of the Congo (DRC) and the Republic of Guinea. The response to these and many previous outbreaks was impeded by weak health systems, complex socio-cultural and political environments, ineffective messaging and community engagement, and civil insecurity. In eastern DRC, the presence of over 100 different armed groups with varied economic and political interests imposed a constant specter of violence ${ }^{1-3}$. Unfortunately, these challenging settings are more the norm than the exception; EVD is a disease of poverty, gaining traction where civil unrest and/or remoteness limit(s) the development of and access to strong health systems ${ }^{4}$. It will never be easy to confront EVD.

\section{Technological advances; implementation challenges}

Recent research advances have produced some effective tools against EVD. These include two vaccines against Ebola virus that have recently received regulatory approval: rVSV-ZEBOV, a single-dose vaccine, made by Merck; and the two-dose Ad26.ZEBOV/ MVA-BN-Filo, made by Janssen Vaccines and Prevention ${ }^{5}$.

But how are these vaccines to be used for optimal impact? EVD poses a conundrum: despite its increasing frequency, EVD remains an extremely rare disease, with fewer than 30 distinct outbreaks and a cumulative total of fewer than 40,000 cases since Ebola virus was first discovered in 1976 (refs. ${ }^{1,5}$ ). Despite a theoretical endemic range that covers a large swath of Central and West Africa, with a population at risk of perhaps a billion people, the

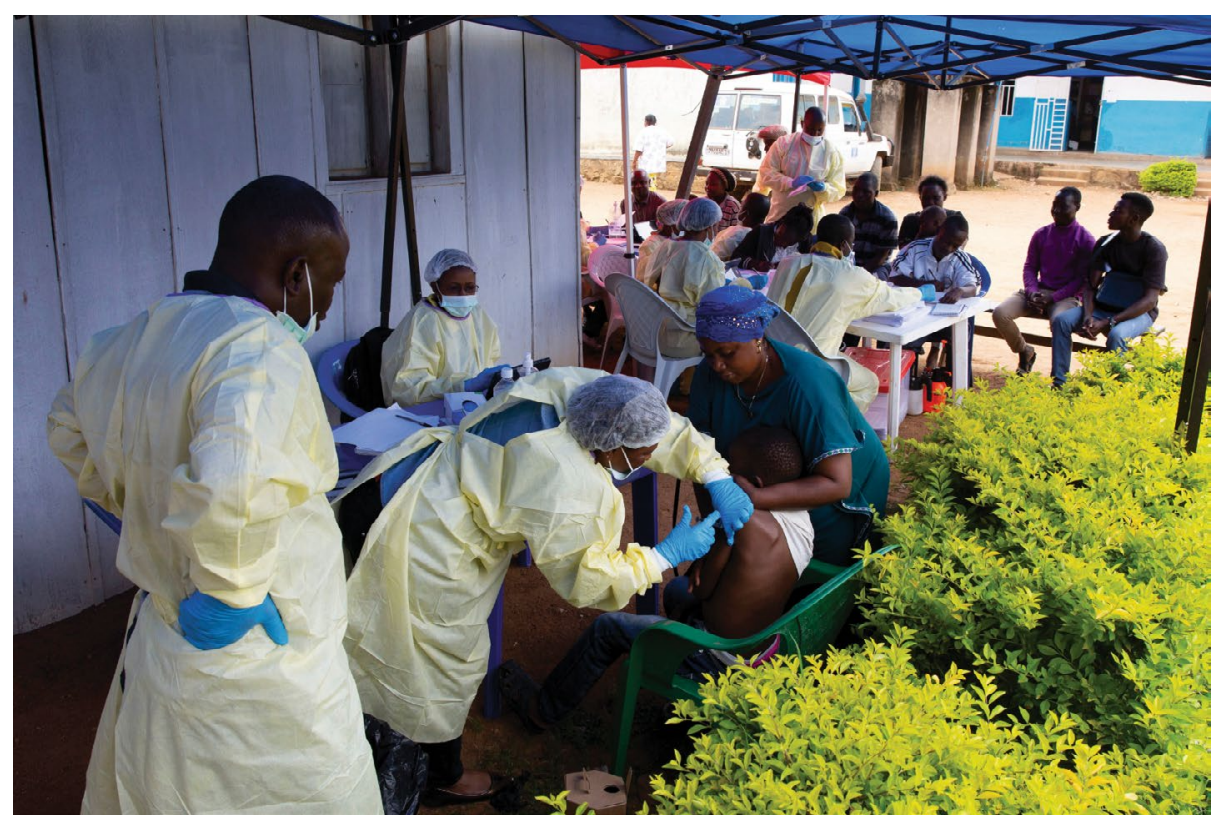

Vaccination campaign among the population in an Ebola treatment center in Beni, DRC. Credit: Andia / Alamy Stock Photo

risk to any given person is infinitesimally small. No cost-benefit analysis could justify widespread inclusion of the vaccine against EVD into routine vaccination of the population at large. However, outbreaks, when they do occur, cause terrible human suffering and the potential for major disruption on national, regional, and even global scales, at a cost of millions or even billions of dollars for response and lost economic growth. The economic burden of the West Africa outbreak is estimated to be over US $\$ 50$ billion ${ }^{6}$.

Some have advocated a 'hotspot' approach, with population-based vaccination in focused regions at risk. However, present understanding of the ecology of EVD is too rudimentary for reliable spatio-temporal predictions of introduction of the virus into humans. Repeated de novo introductions of Ebola virus from the wild in the same geographic area seem to be rare and fluctuating; from 1994 to 2003, at least seven distinct outbreaks occurred in Gabon and neighboring Republic of the Congo, but none have been reported since ${ }^{1,5}$. Furthermore, human behavioral factors, even harder to predict, are probably as important determinants of where large outbreaks occur as the ecological factors are.

The approach so far has been focused on 'ring vaccination' during outbreaks: quickly identifying and vaccinating contacts of people with EVD, as well as contacts of contacts, and healthcare and other frontline workers. Over 16,000 people in Guinea and 300,000 in eastern DRC were vaccinated through this approach, with a much smaller number in the small outbreaks in DRC's remote Équateur province in recent years ${ }^{7,8}$. Although ring vaccination undoubtedly helped to control the transmission of EVD, it is far from a panacea, as indicated by the fact that both these major outbreaks dragged on for years despite vaccination ${ }^{2,9}$. Ring vaccination faces formidable logistical and social challenges to 
effective implementation; foremost among them is that its success is dependent on the ability to identify nearly all cases and contacts-far from a given in the complex socio-cultural context of EVD outbreaks and already a recognized challenge in the two new outbreaks ${ }^{8,10}$. Furthermore, the ring-vaccination approach, which limits eligibility to a relatively small select group, is not always understood and accepted by the community, which exacerbates tensions over equity ${ }^{2}$. What seems like a relatively simple process becomes complicated, and hence ultimately sub-optimal, which results in continued infection and loss of life as smoldering transmission is slowly ground to a halt.

\section{Toward a new vaccination strategy}

The most effective and implementable strategy should combine facets of existing approaches, including the following.

\section{Widespread routine vaccination of} healthcare, frontline workers and other high-risk groups throughout endemic areas for EVD. Modeling studies suggest the efficacy of this approach in curtailing outbreaks ${ }^{11}$. Healthcare workers, who are essential to effective outbreak control, are at enhanced risk, and nosocomial transmission is a major amplifier in most EVD outbreaks. To account for the frequent turnover of personnel, the approach should not be a one-time mass vaccination but instead should be a routine pre-requisite (unless there is a specific medical contraindication) of employment, akin to the requirement for vaccination against other blood-borne diseases, such as hepatitis B. A similar requirement for vaccination should be implemented for personnel deployed to the outbreak from non-endemic countries. Other frontline workers and high-risk groups, such as traditional healers, pharmacists, hunters, and taxi drivers, should also be included. The need to consistently provide vaccination to these high-risk groups over time can help create a stable market and supply chain for vaccines against EVD, with the additional potential benefit of strengthening health systems for other vaccinations.

Geographic- and/or population-based vaccination campaigns during outbreaks. When outbreaks occur, an immediate geographic-and/or population-based vaccination campaign should be mounted. The target population should be based on the latest epidemiologic and spatio-temporal data and modeling and should comprise all ages and genders, including pregnant and lactating women. The advantages of this approach are that it is not dependent on the identification of contacts, it negates the impression of selective entitlement to vaccination, and it provides a degree of herd immunity against later reintroduction of virus through sexual transmission from male survivors, recrudescence, and de novo introduction from wild animals. While the logistical effort and cost, as well as vaccine supply, of mounting this campaign are much greater than they are for ring vaccination, this would probably be offset by the potential to end outbreaks much earlier and to prevent their rekindling through renewed transmission from survivors or introduction from the wild. Furthermore, the costs and effort required would be mitigated both by the relative simplicity of this approach, which obviates the need for complex enumeration, and by the economies of scale, recognizing that the lion's share of the cost and effort comes from establishing the logistics for vaccination, not the number of people subsequently vaccinated.

Targeted vaccination of sex partners of male survivors. It is now recognized that Ebola virus can persist in the semen of surviving males and can be spread to sex partners for months or even years after recovery from acute disease ${ }^{12}$. It is thus essential to identify and vaccinate all sex partners, who can be identified while the male patient is hospitalized and can be vaccinated before he is discharged. Ongoing monitoring will be warranted for the identification and vaccination of new partners.

\section{Expansion of the target population} after the outbreak is declared over. It is reasonable to assume that in the months and years immediately following an outbreak, the virus remains present in the local animal reservoir, and thus the risk of new introductions is elevated. Consideration could thus be given to geographic expansion of the target population. In addition to protecting the population from EVD, this undertaking may again provide an opportunity for general strengthening of the health system, and also for helping to address equity concerns over unequal access. In the long run, ecosystem surveillance for Ebola virus in bats, the suspected reservoir, and other wild-animal intermediate hosts could help to focus preventive vaccination in specific high-risk geographic areas and seasons.
As to which vaccine should be used, there is probably a niche for both the Merck product and the Janssen product, with adaption to the strengths of each in the number of doses and cold chain required, adverse effects, safety in pregnant women and the immunocompromised, ability to boost in the face of vector immunity, and lag time to and duration of immunity. That final point is of particular importance, as the cost-benefit justification for the aforementioned strategy may alter substantially if protection is short-lived. Finally, it should be noted that both vaccines are designed to protect against only the Zaire ebolavirus species of Ebola virus. Expansion of the platforms to cover all pathogenic viruses in the Ebolavirus genus, as well as related Marburg viruses, would underscore the need for broader incorporation of these products into health systems, and the good that can be derived from their judicious use.

\section{Daniel G. Bausch ${ }^{凶}$}

UK Public Health Rapid Support Team, Public Health England/London School of Hygiene \& Tropical Medicine, London, UK.

$\bigotimes_{e-m a i l: d a n i e l . b a u s c h @ l s h t m . a c . u k}$

Published online: 5 April 2021

https://doi.org/10.1038/s41591-021-01313-w

References

1. Bausch, D. G. Curr. Top. Microbiol. Immunol. 411, 63-92 (2017).

2. Bausch, D. G. \& Piot, P. J. Infect. Dis. 222, 521-524 (2020).

3. Kraemer, M. U. G. et al. BMC Med. 18, 113 (2020).

4. Bausch, D.G. \& Clougherty, M.M. J. Infect. Dis. (2015),

5. Feldmann, H., Sprecher, A. \& Geisbert, T. W. N. Engl. J. Med. 382, 1832-1842 (2020).

6. Huber, C., Finelli, L. \& Stevens, W. J. Infect. Dis. 218, S698-S704 (2018)

7. Henao-Restrepo, A. M. et al. Lancet 389, 505-518 (2017).

8. World Health Organization. https://apps.who.int/iris/bitstream/ handle/10665/332654/SITREP_EVD_DRC_20200623-eng. pdf? sequence $=1$ \&is Allowed $=y$ (23 June 2020).

9. Vetter, P. \& Kaiser, L. Rev. Med. Suisse 16, 739-743 (2020). 10. Kucharski, A. J. et al. Emerg. Infect. Dis. 22, 105-108 (2016).

11. Robert, A. et al. Epidemics 27, 106-114 (2019).

12. Bausch, D. G. et al. Lancet Infect. Dis. 16, e82-e91 (2016).

\section{Acknowledgements}

The UK Public Health Rapid Support Team is funded by the UK Department of Health and Social Care. The views expressed in this publication are those of the author and not necessarily those of the National Health System, the National Institute for Health Research or the Department of Health and Social Care. The author thanks R. Grais and S. Nichol for feedback on the manuscript.

\section{Competing interests}

D.G.B. is a co-principle investigator for a phase 3 study of the Ad26.ZEBOV/MVA-BN-Filo vaccine in the DRC. The London School of Hygiene \& Tropical Medicine is the study sponsor for numerous clinical trials of the Ad26. ZEBOV/MVA-BN-Filo vaccine and has also received grant funding for clinical trials of rVSV-EBOV. 